\title{
ACTA2 is not a major disease-causing gene for moyamoya disease
}

\author{
Journal of Human Genetics (2009) 54, 687-688; doi:10.1038/jhg.2009.91; published online 11 September 2009
}

In 2007, Guo et al. ${ }^{1}$ reported mutations of the vascular smooth muscle cell-specific isoform of $\alpha$-actin (ACTA2), a major component of the contractile apparatus in smooth muscle cells located throughout the arterial system, in patients with thoracic aortic aneurysms and dissections (TAAD). This is the second responsible gene for familial TAAD, and the first is the $\beta$-myosin heavy chain $\left(\right.$ MYH11). ${ }^{2,3}$ Subsequently, in 2009, Guo et al. ${ }^{4}$ reported again that the mutations of ACTA2 caused not only familial TAAD but also a diversity of vascular disease, including premature onset of coronary artery disease and premature ischemic strokes. Interestingly, moyamoya disease (MMD) was also included in this variety derived from ACTA2 mutations. Briefly, two families with ACTA2 mutations, TAA105 with p.R258H and TAA390 with p.R258C, who had not been described as having MMD, were reported to have familial MMD. ${ }^{4}$

Moyamoya disease is a specific cerebrovascular disease characterized by stenosis or occlusion of the terminal portions of the internal carotid arteries and the formation of an abnormal vascular network in the vicinity of the arterial occlusion. ${ }^{4}$ In 1969 , this entity was first described in English as 'moyamoya disease'; moyamoya is a Japanese word that means something hazy such as a puff of cigarette smoke, drifting in the air. ${ }^{5}$ Since then, many similar cases have been reported mainly in Japan and Korea, and it turned out to be extremely rare in non-Asian populations. ${ }^{6-8}$ Its prevalence in the entire Japanese population is estimated to be 3 per 100000 persons. ${ }^{9}$ MMD occurs more frequently in females (male-to-female ratio of 2:3) and is prevalent among patients $<10$ years of age. ${ }^{6,9}$ Juvenile patients with MMD initially present with transient motor disturbances - that is, symptoms of transient brain ischemia-whereas adults present with intracranial hemorrhage. The symptoms in juvenile patients are due to the narrowing or occlusion of the circle of Willis, and those in adults are due to a collapse of collateral circulation, which gradually develops as a result of the occlusion of carotid fork at a younger age.

Graham and Matoba $^{10}$ reported that MMD in Hawaii has a higher incidence and prevalence than the rest of the US, mainly because of the larger percentage of Asians, particularly Japanese, living in Hawaii. Regarding the incidence, there was no difference in MMD among Japanese living in Hawaii or Japan. Thus, genetic rather than environmental factors may explain the increased MMD in Hawaii. To reveal the molecular basis of MMD, many studies have been reported to date, including three reports of the whole-genome linkage studies indicating high lod score on chromosome regions of 3p24.2-26, 8q23 and 17q25.3. ${ }^{11-13}$ However, the responsible gene for MMD has not been identified on these regions, and the locus of ACTA2, 10q23.31, has never been reported as a candidate region for Japanese MMD. ${ }^{1,14}$

Guo et al. ${ }^{4}$ reported magnetic resonance angiography findings of TAA105 family members in supplemental figures showing severe stenosis of the terminal internal carotid arteries with extension of the narrowing up to proximal middle cerebral and anterior cerebral arteries. These findings indicate premature stage of MMD, because MMD can be diagnosed when patients showed clinical symptoms derived from neurovascular involvements and their radiographic findings revealed moyamoya vessels as the collateral arteries caused by the stenosis of the region of middle cerebral arteries, as the collateral arteries due to the stenosis of them. ${ }^{15}$ The finding of another patient with TAAD and premature stroke in family TAA377 also indicated premature early stage of MMD in MRA in the supplemental figure reported by Guo et al., but this patient was not diagnosed as having MMD in the report. The patient 8906 in a family sharing p.R212Q mutation was reported as having sporadic MMD with premature stroke. As there were no detailed descriptions about the clinical symptoms of these patients, we do not know whether these patients showed characteristic symptoms of MMD. According to the criteria for MMD, arteriosclerosis should be ruled out. ${ }^{13,15}$ Thus, we cannot determine whether the condition of these patients were really adapted to MMD or not.

Until now, we participated in the studies for the molecular basis of MMD and have published some articles. ${ }^{16-18}$ To confirm whether ACTA2 is a major disease-causing gene for MMD or not, we analyzed the mutations of ACTA2 in Japanese patients with MMD. The subjects were 53 Japanese MMD patients (male/female $=18 / 35$, and 7 patients had familial MMD). By use of the standard PCR-direct sequencing method, we analyzed all coding ACTA2 exons (primers for PCR were originally designed by use of web software primer3; http://frodo.wi.mit. edu/primer $3 /$ ). The result showed no mutation at all.

From this evidence, we think that $A C T A 2$ is not a major responsible gene for MMD, especially for Japanese MMD patients. MMD is an isolated disorder, and there is no previous report of co-existence of MMD and TAAD. As MMD patients usually show ischemic attacks during juvenile period, we think that TAAD derived from ACTA2 mutations would be atypical for MMD. Therefore, further studies are required to identify a major disease-causing gene for MMD. 


\section{ACKNOWLEDGEMENTS}

This work was supported by the Program for Promoting the Establishment of Strategic Research Centers, Special Coordination Funds for Promoting Science and Technology, Ministry of Education, Culture, Sports, Science and Technology (Japan).

Keiko Shimojima and Toshiyuki Yamamoto

International Research and Educational Institute for Integrated Medical Sciences

(IREIIMS), Tokyo Women's Medical University, Tokyo, Japan

E-mail:yamamoto@imcir.twmu.ac.jp

1 Guo, D. C., Pannu, H., Tran-Fadulu, V., Papke, C. L., Yu, R. K., Avidan, N. et al. Mutations in smooth muscle alpha-actin (ACTA2) lead to thoracic aortic aneurysms and dissections. Nat. Genet. 39, 1488-1493 (2007).

2 Zhu, L., Vranckx, R., Khau Van Kien, P., Lalande, A., Boisset, N., Mathieu, F. et al. Mutations in myosin heavy chain 11 cause a syndrome associating thoracic aortic aneurysm/aortic dissection and patent ductus arteriosus. Nat. Genet. 38, 343-349 (2006).
3 Pannu, H., Tran-Fadulu, V., Papke, C. L., Scherer, S., Liu, Y., Presley, C. et al. MYH11 mutations result in a distinct vascular pathology driven by insulin-like growth factor 1 and angiotensin II. Hum. Mol. Genet. 16, 2453-2462 (2007).

4 Guo, D. C., Papke, C. L., Tran-Fadulu, V., Regalado, E. S., Avidan, N., Johnson, R. J. et al. Mutations in smooth muscle alpha-actin (ACTA2) cause coronary artery disease, stroke, and Moyamoya disease, along with thoracic aortic disease. Am. J. Hum. Genet. 84, 617-627 (2009).

5 Suzuki, J. \& Takaku, A. Cerebrovascular 'moyamoya' disease. Disease showing abnormal net-like vessels in base of brain. Arch. Neurol. 20, 288-299 (1969).

6 Suzuki, J. Moyamoya Disease (Springer-Verlag, Berlin, 1983).

7 Goto, Y. \& Yonekawa, Y. Worldwide distribution of moyamoya disease. Neurol. Med. Chir. (Tokyo) 32, 883-886 (1992).

8 Kudo, T. Spontaneous Occlusion of the Circle of Willis (Springer-Verlag, Berlin, 1993).

9 Wakai, K., Tamakoshi, A., Ikezaki, K., Fukui, M., Kawamura, T., Aoki, R. et al. Epidemiological features of moyamoya disease in Japan: findings from a nationwide survey. Clin. Neurol. Neurosurg. 99 (Suppl 2), S1-S5 (1997).

10 Graham, J. F. \& Matoba, A. A survey of moyamoya disease in Hawaii. Clin. Neurol. Neurosurg. 99 (Suppl 2), S31-S35 (1997).

11 Ikeda, H., Sasaki, T., Yoshimoto, T., Fukui, M. \& Arinami, T. Mapping of a familial moyamoya disease gene to chromosome 3p24.2-p26. Am. J. Hum. Genet. 64, 533-537 (1999).
12 Sakurai, K., Horiuchi, Y., Ikeda, H., Ikezaki, K., Yoshimoto, T., Fukui, M. et al. A novel susceptibility locus for moyamoya disease on chromosome 8q23. J. Hum. Genet. 49, 278-281 (2004).

13 Mineharu, Y., Liu, W., Inoue, K., Matsuura, N., Inoue, S., Takenaka, K. et al. Autosomal dominant moyamoya disease maps to chromosome 17q25.3. Neurology 70, 2357-2363 (2008).

14 Ueyama, H., Inazawa, J., Ariyama, T., Nishino, H., Ochiai, Y., Ohkubo, I. et al. Reexamination of chromosomal loci of human muscle actin genes by fluorescence in situ hybridization. Jpn J. Hum. Genet. 40, 145-148 (1995).

15 Fukui, M. Guidelines for the diagnosis and treatment of spontaneous occlusion of the circle of Willis ('moyamoya' disease). Research Committee on Spontaneous Occlusion of the Circle of Willis (Moyamoya Disease) of the Ministry of Health and Welfare, Japan. Clin. Neurol. Neurosurg. 99 (Suppl 2), S238-S240 (1997).

16 Yamamoto, T., Akasaka, Y., Ohtani, K., Hayashi, T., Kashiwagi, S., Ichiyama, T. et al. No association between moyamoya disease and polymorphism of IGF2R. No To Hattatsu 37, 15-19 (2005).

17 Yamamoto, T., Akasaka, Y., Ohtani, K., Hayashi, T., Kashiwagi, S., Ichiyama, T. et al. Molecular screening for moyamoya disease by use of expressed sequence tag on chromosome 3p. No To Hattatsu 37, 20-25 (2005).

18 Paez, M. T. \& Yamamoto, T. Single nucleotide polymorphisms of tissue inhibitor of metalloproteinase genes in familial moyamoya disease. Neurosurgery 60, E582 (2007). 